\title{
Brachial-Ankle Pulse Wave Velocity is Increased and Associated with Disease Activity in Patients with Takayasu Arteritis
}

\author{
Zhiguang Wang ${ }^{1}$, Aimin Dang ${ }^{2}$ and Naqiang Lv ${ }^{2}$
}

\begin{abstract}
${ }^{1}$ Henan Provincial People's Hospital, Fuwai Central China Cardiovascular Hospital, People's Hospital of Zhengzhou University, School of Clinical Medicine, Henan University, Zhengzhou, Henan, China.

${ }^{2}$ Department of Special Care Center, Department of Cardiology, Fuwai Hospital, Chinese Academy of Medical Sciences and Peking Union Medical College, Beijing, China.
\end{abstract}

Aim: Takayasu arteritis (TAK) is a rare inflammatory large-vessel vasculitis with increased cardiovascular morbidity and mortality. Brachial-ankle pulse wave velocity (ba-PWV) is a widely used measure of arterial stiffness and serves as an indicator of either cardiovascular risk or severity of vascular damage. However, the studies about the relationship between TAK and ba-PWV are limited. This study aimed to investigate the use of ba-PWV in the patients with TAK.

Methods: Sixty-seven patients with TAK and 67 age and sex matched healthy controls were recruited. Patients with TAK were grouped according to disease activity. The routine hematological parameters and ba-PWV were summarized.

Results: Ba-PWV was significantly higher in the patients with TAK than in the healthy controls $(P<0.001)$. Ba-PWV was significantly higher in the patients with active TAK than in the patients with inactive TAK $(P=$ 0.04). Multiple liner regression analysis indicated that TAK $(\beta=363.97, P=0.013)$, and mean arterial pressure (MAP) $(\beta=8.52, P=0.012)$ were independently related to ba-PWV. Ba-PWV did not correlate with C-reactive protein (CRP) and erythrocyte sedimentation rate (ESR) in overall patients with TAK (both $P>0.05)$. In patients with TAK without immunosuppressive therapy, ba-PWV significantly correlated with CRP $(r=0.419$, $P=0.008)$ but not ESR $(P>0.05)$. Multiple logistic regression analysis indicated that ba-PWV was an independent predictor of active TAK in overall patients with TAK $(\mathrm{OR}=1.003,95 \% \mathrm{CI}=1.000-1.007 ; P=0.040)$ and patients with TAK without immunosuppressive therapy $(\mathrm{OR}=1.006,95 \% \mathrm{CI}=1.001-1.012 ; P=0.031)$.

Conclusions: Being significantly increased in patients with TAK, ba-PWV is significantly associated with TAK disease activity, and it probably correlates with systematic inflammation.

Key words: Takayasu arteritis, Brachial-ankle pulse wave velocity, Disease activity, Arterial stiffness, Inflammation

\section{Introduction}

Takayasu arteritis (TAK) is a rare, chronic, and non-specific vasculitis that causes inflammation of arteries. Although all large arteries can be affected, the aorta, subclavian, and carotid arteries are most commonly involved $(60 \%-90 \%)^{1,2)}$. Individuals of any race, gender, or age may be affected by TAK, but it is most common in young Asian females in the second and third decades of life. This disease is characterized by adventitial thickening and cellular infiltration of the tunica media, with local destruction of vascular smooth muscle cells and elastin ${ }^{3)}$. It causes blood-vessel wall thickening, dilation or aneurysm, arterial stenosis, and progressive occlusion, resulting in hypertension, ischemia, aortic regurgitation, and absent or reduced pulses. TAK is associated with a significantly increased risk of cardiovascular diseases ${ }^{4-6}$.

Arterial stiffness is an important and independent predictor of future cardiovascular events and all-

Address for correspondence: Aimin Dang, Department of Special Care Center, Department of Cardiology, Fuwai Hospital, No.167 Beilishi Road, 100037 Xicheng District, Beijing, China E-mail: aimindang@fuwaihospital.org

Received: December 11, 2018 Accepted for publication: May 27, 2019

Copyright@2019 Japan Atherosclerosis Society

This article is distributed under the terms of the latest version of CC BY-NC-SA defined by the Creative Commons Attribution License. 
cause mortality ${ }^{7}$, and it is also an indicator of severity of vascular damage ${ }^{8,9)}$. Among the representative measures of arterial stiffness, brachial-ankle pulse wave velocity (ba-PWV) ${ }^{8,10,11)}$, which reflects the stiffness of both the central and peripheral muscular arteries, has been widely used as a simple and non-invasive measure for arterial stiffness.

The increase of arterial stiffness is age-related and may be exacerbated by a number of disorders ${ }^{7,8,12)}$. Inflammation typically plays an important role in arteries stiffening, related to atherosclerosis, arteriosclerosis, endothelial dysfunction, smooth muscle cell migration, vascular calcification, increased activity of metalloproteinases, extracellular matrix degradation, oxidative stress, etc ${ }^{13)}$. In patients with TAK characterized by the vasculitis causing inflammation of the aorta and its main branches, inflammation may dramatically contribute to arterial stiffening. Arterial stiffness may be an important risk factor with important clinical application value for patients with TAK. Previous studies demonstrated that the arterial stiffness, measured as carotid-femoral pulse wave velocity (cfPWV), was increased in patients with TAK ${ }^{14,15)}$. However, these studies are limited because of their small sample sizes of patients with TAK $\left(10^{14}\right)$ and $27^{15)}$ patients with TAK). Moreover, the use of cf-PWV as a measurement of arterial stiffness in the patients with TAK is probably not appropriate because of its low reproducibility. Therefore, at present, the utility of arterial stiffness measured by PWV in the patients with TAK is far from well-established.

\section{Aims}

In this current study, we presented the clinical data of 67 patients with TAK and 67 healthy female subjects who received ba-PWV measurement. Our aim was to evaluate the utility of ba-PWV to measure the arterial stiffness in the patients with TAK, to determine its relationship with TAK, and to investigate its relationships with inflammation as well as TAK disease activity in a relatively large number of patients with TAK.

\section{Methods}

\section{Subjects}

A total of 67 Chinese Han patients with TAK, diagnosed according to American College of Rheumatology criteria ${ }^{16)}$, were enrolled in this study. A total of 67 healthy unrelated age- and gender-matched subjects without any history of chronic disease were used as controls. The study was approved by the Ethics Committee of FuWai Hospital, and all of the subjects gave informed consent. Patients with ankle brachial index $(\mathrm{ABI})<0.9$ were excluded, because ba-PWV was decreased in the presence of arterial occlusive disease in the lower extremities. The higher values of blood pressure were used for the patients with TAK with significant left and right difference of the brachial artery blood pressure, because the lower brachial artery blood pressure may be associated with a significant angiostegnosis. Immunosuppressive therapy refers to treatment with prednisolone or azathioprine in patients with TAK. Disease duration of TAK was between 1 and over 30 years in this study.

\section{Laboratory Assays}

Blood samples were collected after the patients fasted overnight. Total cholesterol, high-density lipoprotein cholesterol (HDL-C), low-density lipoprotein cholesterol (LDL-C), erythrocyte sedimentation rate (ESR), and C-reactive protein (CRP) were measured using routine laboratory methods.

\section{Ba-pwv Measurement}

Ba-PWV were measured after 5 min of bed rest in a quiet and temperature-controlled room $\left(20^{\circ} \mathrm{C}\right.$ to $25^{\circ} \mathrm{C}$ ), with the participant in a supine position. Blood pressure, ba-PWV, ABI, and electrocardiogram were simultaneously recorded using a validated oscillometric device (Model BP203RPE-II [VP-1000], Omron Corporation, Japan ${ }^{17)}$. In this study, the average values of the right and left ba-PWV were used for analysis. Height and body weight were obtained and recorded using an oscillometric device, with the body mass index (BMI) calculated and recorded. Heart rate (HR), right arm systolic blood pressure (SBP), right arm diastolic blood pressure (DBP), and mean arterial pressure (MAP) were recorded. Pulse pressure (PP) was obtained as the difference between the right arm SBP and right arm DBP.

\section{Statistical Analyses}

Continuous variables are presented as mean \pm standard deviation. Categorical variables were expressed as percentages. Comparisons of baseline characteristics were conducted using the Student's $t$-test for normally distributed variables, Mann-Whitney $U$ test for significantly skewed continuous variables, and chi-square $\left(\chi^{2}\right)$ tests for categorical variables. Univariate analyses were initially applied to test the associations between ba-PWV and other variables. Correlations between skewed continuous or discrete variables were evaluated using Spearman's r coefficient, whereas those between normally distributed variables were evaluated using Pearson's $r$ coefficient. Simple linear regression analyses were used to evaluate the 
Table 1. Basic characteristics of healthy subjects and patients with TAK

\begin{tabular}{|c|c|c|}
\hline Variable & Healthy subjects $(N=67)$ & TAK patients $(N=67)$ \\
\hline Age (years) & $39.67 \pm 9.29$ & $35.68 \pm 10.42^{*}$ \\
\hline Female sex $(\%)$ & 100 & 100 \\
\hline BMI $\left(\mathrm{kg} / \mathrm{m}^{2}\right)$ & $25.54 \pm 3.08$ & $24.00 \pm 4.42$ \\
\hline Diabetes(\%) & 0 & $4.4^{*}$ \\
\hline Aspirin (\%) & 0 & $28.8^{*}$ \\
\hline Statins (\%) & 0 & $7.6^{*}$ \\
\hline Immunosuppression (\%) & 0 & $34.7^{*}$ \\
\hline ARB /ACEI (\%) & 0 & $22.8^{*}$ \\
\hline CCB (\%) & 0 & $22.9^{*}$ \\
\hline$\beta$-blocker (\%) & 0 & $30.4^{*}$ \\
\hline Diuretics (\%) & 0 & $6.3^{*}$ \\
\hline Heart rate (beats/min) & $68.21 \pm 11.04$ & $78.16 \pm 11.94^{*}$ \\
\hline $\mathrm{SBP}(\mathrm{mmHg})$ & $117.70 \pm 11.29$ & $122.51 \pm 32.08$ \\
\hline $\mathrm{DBP}(\mathrm{mmHg})$ & $69.78 \pm 9.21$ & $67.93 \pm 19.87$ \\
\hline MAP (mmHg) & $87.97 \pm 9.48$ & $90.43 \pm 24.12$ \\
\hline PP $(\mathrm{mmHg})$ & $47.91 \pm 8.03$ & $32.09 \pm 12.65^{* *}$ \\
\hline $\operatorname{ESR}(\mathrm{mm} / \mathrm{h})$ & $6.84 \pm 8.57$ & $13.97 \pm 15.88^{* *}$ \\
\hline CRP (mg/L) & $3.59 \pm 3.80$ & $6.54 \pm 12.26^{* *}$ \\
\hline Total cholesterol (mmol/L) & $4.72 \pm 0.92$ & $4.40 \pm 0.91^{*}$ \\
\hline HDL-C (mmol/L) & $1.24 \pm 0.28$ & $1.39 \pm 0.34$ \\
\hline $\mathrm{LDL}-\mathrm{C}(\mathrm{mmol} / \mathrm{L})$ & $2.90 \pm 0.82$ & $2.41 \pm 0.76^{*}$ \\
\hline ba-PWV (cm/s) & $1211.37 \pm 154.42$ & $1495.55 \pm 431.72^{*}$ \\
\hline $\mathrm{ABI}$ & $1.14 \pm 0.09$ & $1.22 \pm 0.22$ \\
\hline
\end{tabular}

$\mathrm{ABI}=$ ankle brachial index; $\mathrm{ARB}=$ angiotensin II receptor blocker; $\mathrm{ACEI}=$ angiotensin-converting enzyme inhibitor; ba$\mathrm{PWV}=$ brachial-ankle pulse wave velocity; $\mathrm{BMI}=$ body mass index; $\mathrm{CCB}=$ calcium channel blocker; $\mathrm{CRP}=\mathrm{c}$-reactive protein; $\mathrm{DBP}=$ diastolic blood pressure; $\mathrm{ESR}=$ erythrocyte sedimentation rate; HDL-C=high-density lipoprotein cholesterol; $\mathrm{LDL}-\mathrm{C}=$ low-density lipoprotein cholesterol; $\mathrm{MAP}=$ mean arterial blood pressure; $\mathrm{PP}=$ pulse pressure; $\mathrm{SBP}=$ systolic blood pressure; TAK = Takayasu Arteritis.

Healthy subjects vs. Patients with TAK: ${ }^{*} p<0.05,{ }^{* *} p<0.001$.

associations between ba-PWV and other variables. Multiple linear regression analyses were performed to evaluate the relationships between ba-PWV and TAK while adjusting for potential confounders. A multivariable logistic regression model was performed to identify potential predictive factors for patients with active TAK. CRP and ESR were log-transformed. Data were analyzed using Statistical Package for Social Sciences (SPSS) software (version 13.0, SPSS Inc., Chicago, IL, USA). All statistical tests were two sided. $P<0.05$ was considered to indicate significant difference.

\section{Results}

\section{Patient Characteristics}

The basic characteristics of the study populations are summarized in Table 1 . The basic characteristics of the healthy subjects and the patients with active or inactive TAK are summarized in Table 2. The healthy subjects and patients with TAK were age and sex matched.

No difference of BMI $(25.54 \pm 3.08$ vs. $24.00 \pm$ $\left.4.42 \mathrm{~kg} / \mathrm{m}^{2}\right)$, SBP $(117.70 \pm 11.29$ vs. $122.51 \pm 32.08$ $\mathrm{mmHg})$, DBP $(69.78 \pm 9.21$ vs. $67.93 \pm 19.87$ $\mathrm{mmHg})$, MAP $(87.97 \pm 9.48$ vs. $90.43 \pm 24.12$ $\mathrm{mmHg})$, HDL-C $(1.24 \pm 0.28$ vs. $1.39 \pm 0.34 \mathrm{mmol} /$ $\mathrm{L})$, and $\mathrm{ABI}(1.14 \pm 0.09$ vs. $1.22 \pm 0.22)$ were found between the healthy subjects and the patients with TAK (all $P>0.05)$. Age $(39.67 \pm 9.29$ vs. $35.68 \pm$ 10.42 years, $P<0.05)$, PP $(47.91 \pm 8.03$ vs. $32.09 \pm$ $12.65 \mathrm{mmHg}, P<0.001)$, Total cholesterol (4.72 \pm 0.92 vs. $4.40 \pm 0.91 \mathrm{mmol} / \mathrm{L}, P<0.05)$, and LDL-C $(2.90 \pm 0.82$ vs. $2.41 \pm 0.76 \mathrm{mmol} / \mathrm{L}, P<0.05)$ were significantly higher in the healthy subjects than in the patients with TAK. HR $(68.21 \pm 11.04$ vs. $78.16 \pm$ 11.94 beats $/ \mathrm{min}, P<0.001$ ) was significantly lower in the healthy subjects than in the patients with TAK (Table 1).

\section{TAK and ba-PWV}

Ba-PWV was significantly higher in the patients 
Table 2. Basic characteristics of healthy subjects, inactive and active TAK patients

\begin{tabular}{|c|c|c|c|}
\hline Variable & Healthy subjects $(N=67)$ & Inactive TAK patients $(\mathrm{N}=24)$ & Active TAK patients $(\mathrm{N}=43)$ \\
\hline Age (years) & $39.67 \pm 9.29$ & $42.77 \pm 10.09$ & $31.72 \pm 10.59^{\dagger, *}$ \\
\hline Female sex (\%) & 100 & 100 & 100 \\
\hline BMI $\left(\mathrm{kg} / \mathrm{m}^{2}\right)$ & $25.54 \pm 3.08$ & $24.05 \pm 3.23$ & $23.97 \pm 4.95$ \\
\hline Diabetes(\%) & 0 & $4.1^{*}$ & $4.6^{\dagger}$ \\
\hline Aspirin (\%) & 0 & $13.6^{*}$ & $37.2^{\dagger, 末 \neq}$ \\
\hline Statins (\%) & 0 & $4.5^{*}$ & $9.3^{\dagger}$ \\
\hline Immunosuppression (\%) & 0 & $13.6^{*}$ & $46.5^{\dagger}$, \\
\hline ARB /ACEI (\%) & 0 & $13.6^{*}$ & $27.9^{\dagger}$ \\
\hline CCB (\%) & 0 & $18.2^{*}$ & $25.6^{\dagger}$ \\
\hline$\beta$-blocker (\%) & 0 & $18.2^{*}$ & $37.2^{\dagger}$ \\
\hline Diuretics (\%) & 0 & $9.1^{*}$ & $4.7^{\dagger}$ \\
\hline Heart rate (beats/min) & $68.21 \pm 11.04$ & $75.45 \pm 13.10^{*}$ & $79.67 \pm 11.26^{\dagger}$ \\
\hline $\mathrm{SBP}(\mathrm{mmHg})$ & $117.70 \pm 11.29$ & $116.77 \pm 28.37$ & $125.72 \pm 35.39$ \\
\hline DBP (mmHg) & $69.78 \pm 9.21$ & $63.82 \pm 13.04$ & $70.23 \pm 22.76$ \\
\hline MAP (mmHg) & $87.97 \pm 9.48$ & $86.82 \pm 18.80$ & $92.44 \pm 26.58$ \\
\hline PP $(\mathrm{mmHg})$ & $47.91 \pm 8.03$ & $29.95 \pm 13.03^{*}$ & $33.28 \pm 12.43^{\dagger \dagger}$ \\
\hline $\operatorname{ESR}(\mathrm{mm} / \mathrm{h})$ & $6.84 \pm 8.57$ & $7.59 \pm 4.20$ & $17.23 \pm 18.52^{\dagger, \ddagger}$ \\
\hline $\mathrm{CRP}(\mathrm{mg} / \mathrm{L})$ & $3.59 \pm 3.80$ & $2.65 \pm 1.65$ & $8.53 \pm 14.69^{\dagger, *}$ \\
\hline Total cholesterol (mmol/L) & $4.72 \pm 0.92$ & $4.12 \pm 0.90$ & $4.55 \pm 0.93$ \\
\hline HDL-C (mmol/L) & $1.24 \pm 0.28$ & $1.29 \pm 0.33^{*}$ & $1.44 \pm 0.34$ \\
\hline LDL-C (mmol/L) & $2.90 \pm 0.82$ & $2.31 \pm 0.84^{*}$ & $2.47 \pm 0.71$ \\
\hline ba-PWV (cm/s) & $1211.37 \pm 154.42$ & $1,381.75 \pm 373.33^{* *}$ & $1,553.72 \pm 451.76^{\dagger, 末}$ \\
\hline $\mathrm{ABI}$ & $1.14 \pm 0.09$ & $1.23 \pm 0.24$ & $1.22 \pm 0.21$ \\
\hline
\end{tabular}

$\mathrm{ABI}=$ ankle brachial index; $\mathrm{ARB}=$ angiotensin II receptor blocker; $\mathrm{ACEI}=$ angiotensin-converting enzyme inhibitor; ba-PWV $=$ brachialankle pulse wave velocity; $\mathrm{BMI}=$ body mass index; $\mathrm{CCB}=$ calcium channel blocker; $\mathrm{CRP}=\mathrm{c}$-reactive protein; $\mathrm{DBP}=$ diastolic blood pressure; $\mathrm{ESR}=$ erythrocyte sedimentation rate; HDL-C=high-density lipoprotein cholesterol; LDL-C=low-density lipoprotein cholesterol; $\mathrm{MAP}=$ mean arterial blood pressure; $\mathrm{PP}=$ pulse pressure; $\mathrm{SBP}=$ systolic blood pressure; $\mathrm{TAK}=$ Takayasu Arteritis.

Healthy subjects vs. Inactive TAK patients: ${ }^{*} P<0.05,{ }^{* *} P<0.001$.

Healthy subjects vs. Active TAK patients: ${ }^{\top} P<0.05,{ }^{\top} P<0.001$.

Inactive TAK patients vs. Active TAK patients: ${ }^{*} P<0.05,{ }^{*} P<0.001$.

with TAK than in the healthy subjects $(1495.55 \pm$ 431.72 vs. $1211.37 \pm 154.42 \mathrm{~cm} / \mathrm{s}, P<0.05)$ (Table 1$)$, and it was also significantly higher in the patients with inactive TAK than in the healthy subjects $(1,381.75 \pm$ 373.33 vs. $1211.37 \pm 154.42 \mathrm{~cm} / \mathrm{s}, P<0.001$ ) (Table 2 ; Fig. 1).

Simple linear regression analysis demonstrated that ba-PWV was significantly associated with TAK ( $\beta$ $=214.70, P<0.001$ ) (Table 3 ). In the multiple linear regression analysis using ba-PWV as dependent variable, TAK $(\beta=363.97, P=0.013)$, and MAP $(\beta$ $=8.52, P=0.012$ ) were significantly associated with ba-PWV after adjusting for age, SBP, DBP, PP, BMI, HR, Total cholesterol, HDL-C, and LDL-C (all $P>$ 0.05) $\left(R^{2}=0.413\right)($ Table 3).

\section{Inflammation and ba-PWV in Patients with TAK}

ESR $(13.97 \pm 15.88$ vs. $6.84 \pm 8.57 \mathrm{~mm} / \mathrm{h}, P<$ $0.001)$ and CRP $(6.54 \pm 12.26$ vs. $3.59 \pm 3.80 \mathrm{mg} / \mathrm{L}$, $P<0.001)$ were significantly higher in the patients with TAK than in the healthy subjects (Table 1 ). Patients with TAK were classified into patients with active TAK $(n=43)$ or patients with inactive TAK $(n=24)$ according to Kerr's criteria ${ }^{2)}$. ESR (17.23 \pm 18.52 vs. $7.59 \pm 4.20 \mathrm{~mm} / \mathrm{h}, P=0.002)$ and CRP $(8.53 \pm 14.69$ vs. $2.65 \pm 1.65 \mathrm{mg} / \mathrm{L}, P=0.013)$ were also significantly higher in the patients with active TAK than in patients with inactive TAK (Table 2).

No significant associations between ba-PWV and ESR/CRP were found in overall patients with TAK and patients with active TAK or patients with inactive TAK (all $P>0.05$ ).

In view of the significant influence of immunosuppressive therapy on the levels of ESR and CRP, the associations between ba-PWV and ESR/CRP may be significantly affected by the immunosuppressive therapy. Thus, we examined the associations of ba-PWV and ESR/CRP in overall patients with TAK and the patients with $(n=25$, active TAK 20 , inactive TAK 5) or without immunosuppressive therapy $(n=42$, active 


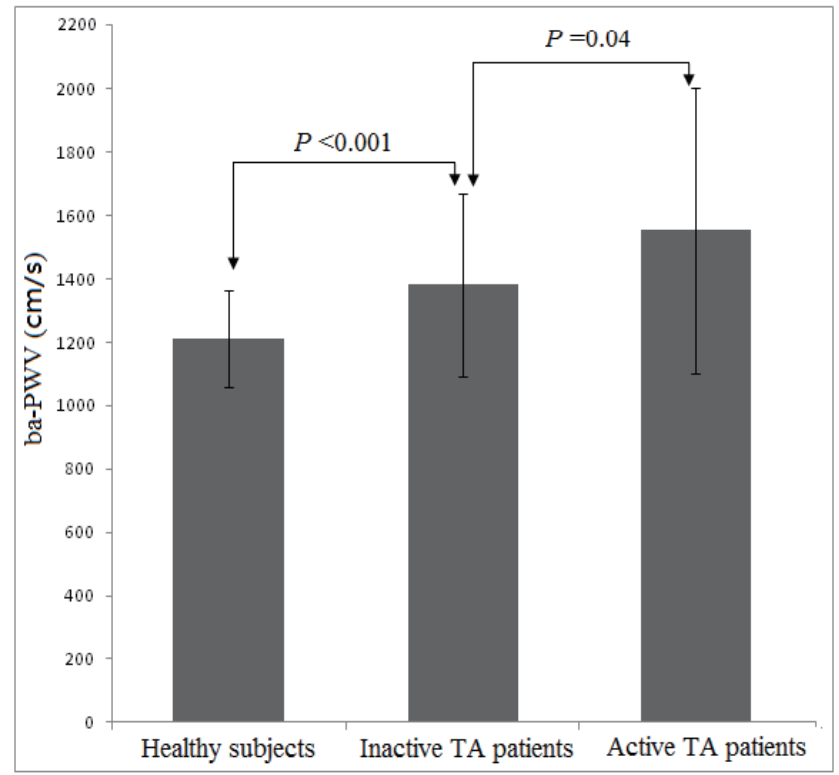

Fig. 1. Ba-PWV of healthy subjects, patients with inactive TAK and patients with active TAK

Ba-PWV was higher in patients with inactive TAK than in healthy subjects but lower than in patients with active TAK.

$\mathrm{CRP}=\mathrm{c}$-reactive protein; $\mathrm{ESR}=$ erythrocyte sedimentation rate; TAK= Takayasu Arteritis.

Table 3. Simple linear regression analysis and multiple linear regression analysis using ba-PWV as dependent variable

\begin{tabular}{|c|c|c|}
\hline Variable & $\beta$ & $P$ value \\
\hline \multicolumn{3}{|l|}{ Simple linear regression analysis } \\
\hline TAK & 214.70 & $<0.001$ \\
\hline Age (years) & -4.18 & 0.040 \\
\hline $\operatorname{BMI}\left(\mathrm{kg} / \mathrm{m}^{2}\right)$ & 6.27 & $>0.05$ \\
\hline Heart rate (beats/min) & 6.96 & $<0.001$ \\
\hline $\mathrm{SBP}(\mathrm{mmHg})$ & 4.86 & $<0.001$ \\
\hline $\mathrm{DBP}(\mathrm{mmHg})$ & 7.45 & $<0.001$ \\
\hline MAP (mmHg) & 2.77 & 0.046 \\
\hline $\mathrm{PP}(\mathrm{mmHg})$ & -2.18 & $>0.05$ \\
\hline $\operatorname{ESR}(\mathrm{mm} / \mathrm{h})$ & 3.84 & 0.044 \\
\hline CRP (mg/L) & 3.76 & $>0.05$ \\
\hline Total cholesterol $(\mathrm{mmol} / \mathrm{L})$ & 18.38 & $>0.05$ \\
\hline HDL-C (mmol/L) & 88.77 & $>0.05$ \\
\hline LDL-C (mmol/L) & -8.14 & $>0.05$ \\
\hline \multicolumn{3}{|l|}{ Multiple linear regression analysis } \\
\hline TAK & 363.97 & 0.013 \\
\hline Age (years) & 3.21 & $>0.05$ \\
\hline $\operatorname{BMI}\left(\mathrm{kg} / \mathrm{m}^{2}\right)$ & 2.04 & $>0.05$ \\
\hline Heart rate (beats/min) & 2.24 & $>0.05$ \\
\hline $\mathrm{SBP}(\mathrm{mmHg})$ & Excluded & - \\
\hline $\mathrm{DBP}(\mathrm{mmHg})$ & 0.53 & $>0.05$ \\
\hline MAP (mmHg) & 8.52 & 0.012 \\
\hline PP (mmHg) & -1.88 & $>0.05$ \\
\hline $\operatorname{ESR}(\mathrm{mm} / \mathrm{h})$ & 1.60 & $>0.05$ \\
\hline $\mathrm{CRP}(\mathrm{mg} / \mathrm{L})$ & -2.40 & $>0.05$ \\
\hline Total cholesterol $(\mathrm{mmol} / \mathrm{L})$ & 71.60 & $>0.05$ \\
\hline HDL-C (mmol/L) & -70.16 & $>0.05$ \\
\hline LDL-C (mmol/L) & -39.99 & $>0.05$ \\
\hline
\end{tabular}

ba-PWV = brachial-ankle pulse wave velocity; $C R P=c$-reactive protein; $E S R=$ erythrocyte sedimentation rate; $\mathrm{TAK}=$ Takayasu Arteritis. 

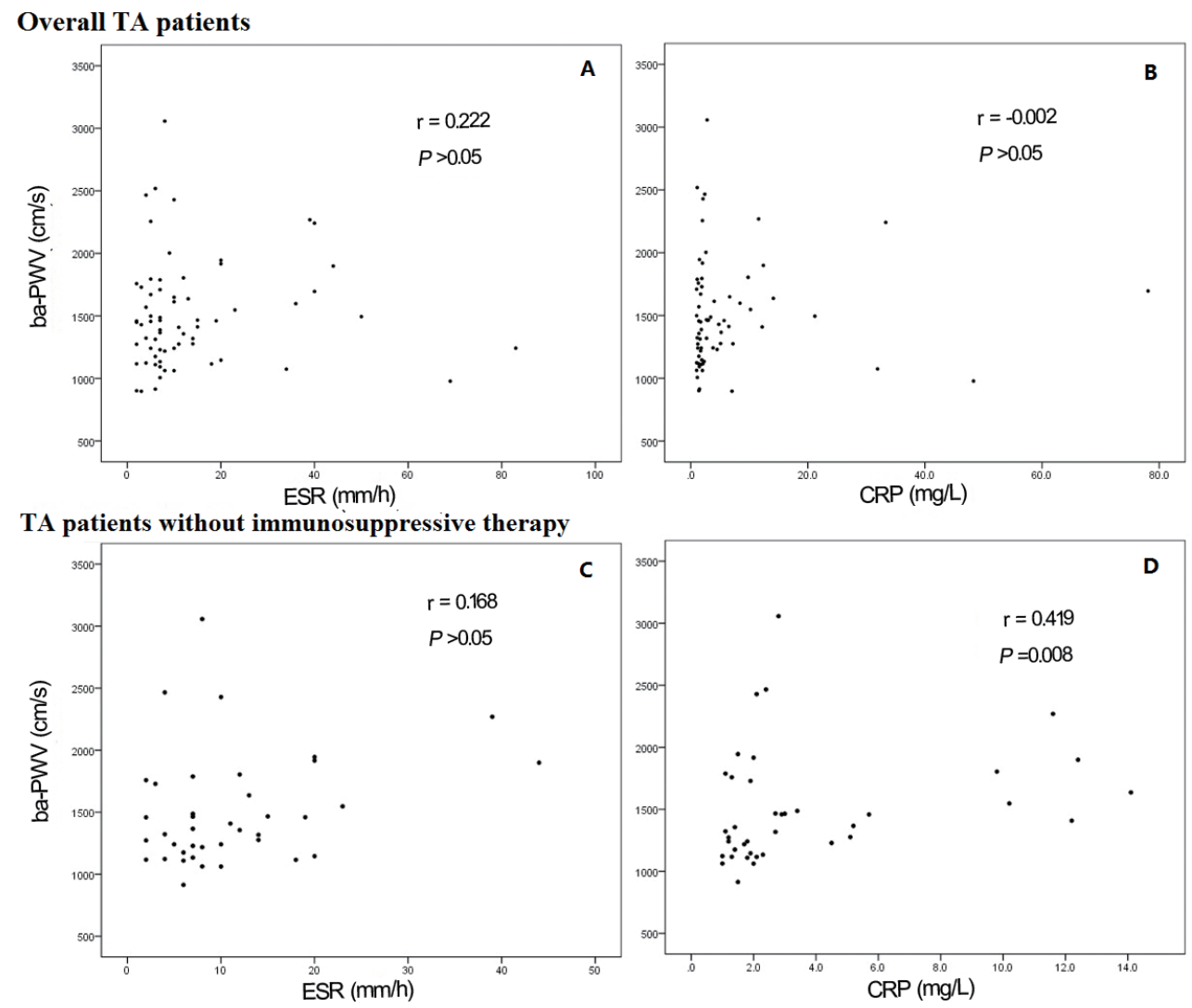

Fig. 2. Relationships between ba-PWV and CRP and ESR in the overall patients with TAK and the patients with TAK without immunosuppressive therapy

Ba-PWV was significantly correlated with CRP in the patients with TAK without immunosuppressive therapy (D) but not in the overall patients with TAK (B). Ba-PWV was not significantly correlated with ESR in the patients with TAK without immunosuppressive therapy $(\mathrm{A})$ and in the overall patients with TAK $(\mathrm{C})$. Correlation coefficient $(\mathrm{r})$ and probability $(\mathrm{P})$ are indicated in each diagram.

TAK 23, inactive TAK 19). In overall patients with TAK and patients with TAK with immunosuppressive therapy, ba-PWV was not significantly correlated with CRP/ESR (all $P>0.05$ ). However, in patients with TAK without immunosuppressive therapy, ba-PWV was significantly correlated with CRP $(r=0.419, P=$ $0.008)$ but not $\operatorname{ESR}(r=0.168, P>0.05)$ (Fig. 2).

\section{Ba-PWV and the Disease Activity of TAK}

Ba-PWV was significantly higher in the patients with active TAK than the patients with inactive TAK $(1,553.72 \pm 451.76$ vs. $1,381.75 \pm 373.33 \mathrm{~cm} / \mathrm{s}, P=$ 0.04) (Table 2; Fig. 1).

We assessed the predictive value of ba-PWV for active TAK with multiple logistic regression analysis (enter method) in overall patients with TAK. In view of the possible influence of immunosuppressive therapy on the analysis result, we also run the analysis in patients with TAK without immunosuppressive therapy. Ba-PWV were significant in the multiple logistic regression analyses for overall patients with TAK (OR $=1.003,95 \% \mathrm{CI}=1.000-1.007 ; P=0.040)$ and patients with TAK without immunosuppressive therapy $(\mathrm{OR}=1.006,95 \% \mathrm{CI}=1.001-1.012 ; P=0.031)$, after adjusting age, SBP, ESR, and CRP. In order to make the results more readable, we used ba-PWV/200 to stand for the ba-PWV divided by 200 in the multiple logistic regression analyses. Ba-PWV/200 were significant in the multiple logistic regression analysis for overall patients with TAK $(\mathrm{OR}=1.915,95 \%$ $\mathrm{CI}=1.032-3.686 ; P=0.040)$ and patients with TAK without immunosuppressive therapy $(\mathrm{OR}=3.344$, 95\% $\mathrm{CI}=1.119-9.991 ; P=0.031)$ after adjusting age, SBP, ESR and CRP (Table 4). ESR and CRP were log-transformed in these logistic analyses. These results suggest that ba-PWV is significantly associated with TAK disease activity, and it may be an independent predictor of active TAK in the patients with TAK. 
Table 4. Multiple logistic regression analyses with active TAK as dependent variable and Age, ESR, ba-PWV/200 and CRP as independent variables

\begin{tabular}{lcc}
\hline Variable & OR $(95 \% \mathrm{CI})$ & $P$ value \\
\hline Overall TAK patients $(n=67)$ & & \\
Age (years) & $0.862(0.788-0.942)$ & 0.001 \\
SBP $(\mathrm{mmHg})$ & $0.995(0.968-1.023)$ & 0.742 \\
ESR $(\mathrm{mm} / \mathrm{h})$ & $30.498(1.390-669.024)$ & 0.030 \\
CRP $(\mathrm{mg} / \mathrm{L})$ & $0.477(0.039-5.911)$ & 0.565 \\
ba-PWV/200 & $1.915(1.032-3.686)$ & 0.040 \\
TAK patients without immunosuppressive therapy $(n=43)$ & & \\
Age (years) & $0.860(0.769-0.961)$ & 0.008 \\
SBP $(\mathrm{mmHg})$ & $0.997(0.958-1.038)$ & 0.890 \\
ESR $(\mathrm{mm} / \mathrm{h})$ & $1.444(1.939-10732.852)$ & 0.024 \\
CRP $(\mathrm{mg} / \mathrm{L})$ & $0.167(0.006-4.874)$ & 0.298 \\
ba-PWV/200 & $3.344(1.119-9.991)$ & 0.031 \\
\hline
\end{tabular}

ba-PWV = brachial-ankle pulse wave velocity; $C R P=c$-reactive protein; $E S R=$ erythrocyte sedimentation rate; $T A K=$ Takayasu Arteritis; $O R=$ odds ratio; $\mathrm{CI}=$ confidence interval. CRP and ESR were log-transformed. ba-PWV/200 refers to ba-PWV divided by 200.

\section{Discussion}

The results in this study suggest that the arterial stiffness measured by ba-PWV is significantly increased in patients with TAK comparing with normal controls, it is also significantly increased in patients with active TAK comparing with patients with non-active TAK, TAK, and MBP is significantly and independently associated with ba-PWV, ba-PWV probably correlates with systematic inflammation evaluated by CRP, and ba-PWV was significantly associated with disease activity in patients with TAK probably serving as an independent predictor of patients with active TAK.

In our study, ba-PWV was higher in patients with TAK with active or inactive disease than in the healthy controls. Moreover, TAK was significantly associated with ba-PWV independently of age, blood pressure, or lipid profile. Two previous studies demonstrated that TAK is associated with higher arterial stiffness, which was measured by cf-PWV and cr-PWV (carotid-radial PWV) in one study ${ }^{14)}$ and cf-PWV in another study ${ }^{15)}$. They have relatively small sample sizes $\left(10^{14)}\right.$ and $27^{15)}$ patients with TAK). The arterial stiffness was demonstrated higher in patients with TAK comparing with normal controls, and it did not correlate with ESR or CRP. One study also demonstrated that TAK and mean BP were the strongest determinants of arterial stiffness measured by cf-PWV, and cf-PWV was not significantly associated with disease activity, history of hypertension, and TAK disease duration ${ }^{15}$.

In our study, we investigated the relationship between ba-PWV and TAK, the relationship between
ba-PWV and TAK disease activity, and the relationship between ba-PWV and systematic inflammation in patients with TAK. We have our own characteristics and strengths as follows. First, there were 67 patients with TAK in our study. In case of the rarity of patients with TAK, our sample size is dramatically large. Second, our study demonstrated a significant association between TAK disease activity and arterial stiffness, and we also initially investigated the relationship between arterial stiffness and systematic inflammation in patients with TAK considering the influence of immunosuppressive therapy. Third, we used ba-PWV as a measurement of arterial stiffness in this study. This is a PWV more widely used in China comparing with cf-PWV. Finally, ba-PWV is probably a better measurement of arterial stiffness for patients with TAK comparing with cf-PWV because cf-PWV has a low reproducibility in patients with TAK.

At present, both cf-PWV and ba-PWV are a widely used measure of arterial stiffness in clinical settings. Cf-PWV was used as a measure of arterial stiffness in the previous two studies ${ }^{14,15)}$, and ba-PWV was used in the present study. Cf-PWV reflects the arterial stiffness in large central arteries between carotid and femoral arteries. Ba-PWV is an automatic PWV measurement reflecting arterial stiffness in the large central and middle-sized peripheral arteries between the brachial artery to ankle artery ${ }^{18)}$.

We believe ba-PWV is more appropriate to be used as a measure of arterial stiffness for patients with TA comparing with cf-PWV. First, more arteries are involved in the measurement of ba-PWV comparing with cf-PWV. TAK frequently affects peripheral arteries, especially subclavian arteries and even axillary 
arteries and femoral arteries, which are involved by baPWV but not by cf-PWV. Furthermore, cf-PWV is not a stable measurement of arterial stiffness for the patients with TAK. It has a low reproducibility in the measurement of arterial stiffness for patients with TAK and that is why the investigators used the average of multiple recordings in the two previous studies (10 recordings in one study ${ }^{14}$ ) and two recordings in another study $\left.{ }^{15}\right)$. The sensors must be located on carotid and femoral arteries in the measurement of cfPWV. However, carotid and femoral arteries are significantly damaged by TAK for many patients with TAK. Therefore, measuring cf-PWV for the patients with TAK comparing with healthy people or patients with other kinds of diseases is a totally different condition. The reproducibility of cf-PWV is low in patients with TAK probably because the sensors cannot be very steadily located on the carotid and femoral arteries, which are very big and significantly affected by TAK. Being involved in atherosclerosis, TAK alters arterial structure, compliance, and functions; thus, it may probably lead to pathological pulsation in carotid and femoral arteries, which may consequently lead to a low reproducibility of cf-PWV in the patients with TAK. Furthermore, obtaining ba-PWV compared with cf-PWV is much easier and convenient. The distance between the carotid and femoral arteries is not easy to be very properly obtained in the measurement of cf-PWV, especially for the subjects with a big abdomen. The iliac region must be exposed before the femoral sensor is located on the femoral artery, in the measurement of cf-PWV. Finally, we already proved the prognostic value of ba-PWV in the patients with Takayasu Arteritis with drug-eluting stent implantation ${ }^{19)}$. Therefore, we think ba-PWV is a more appropriate non-invasive quantification of arterial stiffness for the patients with TAK comparing with cf-PWV.

It is reasonable for the arterial stiffness to be increased in the patients with TAK, which is characterized as an inflammatory granulomatous vasculitis of medium and large arteries and eventually leads to adventitial thickening and cellular infiltration of the tunica media, irregular fibrosis of the blood vessels due to chronic vasculitis, and local destruction of vascular smooth muscle cells and elastin ${ }^{3)}$. The degeneration of elastic fibers is also a feature of TAK, with the formation of aneurysms occurring when inflammation leads to a loss of medial smooth muscle cells. The vascular remodeling consequently contributes to arterial stiffening in patients with TAK. For patients with TAK, the increased arterial stiffness dramatically depends on the inflammatory process, which has been proved to play an important role in arterial stiffening ${ }^{20,21)}$.
Increased arterial stiffness has also been demonstrated in other systemic immune and inflammatory diseases, including Rheumatoid arthritis (RA) ${ }^{22}$, Systemic lupus erythematosus (SLE) ${ }^{23)}$, Wegener's granulomatosis (WG) ${ }^{24)}$, Psoriasis ${ }^{25)}$, Familial Mediterranean fever $(\mathrm{FMF})^{26)}$, Sarcoidosis ${ }^{27)}$, Behçet's disease $(\mathrm{BD})^{28)}$, and systemic sclerosis $(\mathrm{SS})^{29)}$.

Inflammation is a very important contributor in the development of TAK and arterial stiffening. ESR and CRP are widely accepted inflammatory factors, and they also are two important markers monitoring TAK disease activity widely used in clinical settings. In the previous study, the researchers demonstrated that arterial stiffness measured as cf-PWV was not associated with TAK disease activity, ESR, and CRP ${ }^{14,15)}$, but they were significantly limited by their small sample sizes and did not consider the influence of immunosuppressive therapy.

In our study, we examined the relationships between ba-PWV and ESR/CRP in the patients with TAK. In view of the significant influence of immunosuppressive therapy on inflammation in the patients with TAK, we examined the relationships between baPWV and ESR/CRP in overall patients with TAK and patients with TAK with and without immunosuppressive therapy. In overall patients with TAK and patients with TAK with immunosuppressive therapy, ba-PWV was not significantly correlated with CRP and ESR. However, in the patients with TAK without immunosuppressive therapy, ba-PWV was significantly correlated with CRP. Therefore, the significant correlation between ba-PWV and CRP in patients with TAK was probably compromised by the immunosuppressive therapy. Even though more studies are needed, our results suggest that ba-PWV is correlated with systematic inflammation evaluated by CRP in the patients with TAK.

The disease activity of the patients with TAK is a very important concern for physicians in their clinical work. Therefore, the association between disease activity and arterial stiffness is an important topic. In our study, we, for the first time, demonstrated the association between disease activity and arterial stiffness measured as ba-PWV in patients with TAK. We found that although active patients with TAK were much younger than inactive patients with TAK in this study, ba-PWV was significantly higher in patients with active TAK than in patients with inactive TAK. The multiple logistic regression analyses indicated that baPWV was an independent predictor of patients with active TAK in overall patients with TAK and patients with TAK without immunosuppressive therapy. These results suggest that ba-PWV is significantly associated with disease activity in patients with TAK, and ba- 
PWV probably is an independent predictor of the active TAK. Therefore, ba-PWV may be a potential marker to improve the assessment of TAK disease activity in clinical settings.

In order to appropriately follow the illness state, determine the vascular response to therapy, or make appropriate treatment plans for patients with TAK, correctly assessing TAK disease activity in clinical practice is important. At present, the assessment of TAK disease activity is still controversial $0^{30,31)}$. Combined with other widely used markers, ba-PWV may probably improve the non-invasive assessment of disease activity in clinical settings. As a predictor of cardiovascular risk at the same time, ba-PWV may be used as a maker to improve the selection of high-risk patients with TAK for prophylactic treatment or more regular medical examinations, or it can be used as a treatment target to improve the prognosis of patients with TAK.

The significant association between ba-PWV and inflammation as well as disease activity in patients with TAK may suggest that the increased arterial stiffness may be reversible. The effective control of inflammation and the remission of active TAK may be effective in reducing arterial stiffness and therefore cardiovascular risk for patients with TAK.

The significant associations between arterial stiffness with inflammation and disease activity have also been demonstrated in other systemic immune and inflammatory diseases. One study demonstrated that ANCA-associated vasculitis was associated with increased arterial stiffness measured as PWV, and the arterial stiffness also was associated with CRP and the disease activity ${ }^{32)}$. Another study demonstrated that anti-tumor necrosis factor-alpha therapy reduced aortic inflammation, and this effect correlated with the decrease in aortic stiffness in RA patients ${ }^{33}$. Some previous studies demonstrated that the control of inflammation by NF- $\alpha$ antagonist was effective to reduce arterial stiffness in $\mathrm{RA}^{21,34)}$.

This study has several limitations as follows. First, we found a significant correlation between baPWV and CRP in patients with TAK without immunosuppressive therapy, but we did not find a significant relationship between ba-PWV and ESR in this study. This is probably because ESR is not a consistently reliable inflammatory marker in patients with TAK. ESR and CRP are both widely used markers of inflammation. However, previous studies showed that ESR is not a consistently reliable surrogate marker of disease activity in patients with $\mathrm{TAK}^{2,35,36)}$. ESR does not change as rapidly as does CRP, either at the start of inflammation or as it goes away, and CRP is a better marker of inflammation because it is not affected by as many other factors as is ESR such as food. In addition, we did not obtain the detailed imaging information about the arteries affected by TAK in the patients with TAK. Moreover, if we have more patients with TAK without any immunosuppressive therapy, the results will be more convincing. Finally, this is a cross-sectional study, and a prospective study concerning the relationship between baPWV and disease activity in the patients with TAK will be more convincing. Further studies about the influence of immunomodulatory drugs on ba-PWV in patients with TAK are needed.

\section{Conclusions}

In conclusion, the arterial stiffness measured by ba-PWV is significantly increased in patients with TAK, possibly correlates with systematic inflammation, and it is significantly associated with TAK disease activity probably serving as an independent predictor of active TAK. As an independent predictor of cardiovascular risk at the same time and combined with other markers, ba-PWV can probably improve the non-invasive assessment of the high-risk patients with TAK in clinic settings.

\section{Acknowledgments}

None.

\section{Conflicts of Interest}

The authors have declared no conflicts of interest.

\section{Notice of Grant Support}

This work was supported by the National Natural Science Foundation of China (NO. 81170285) and Henan Province Key Research and Generalization Project (NO. 182102311216).

\section{References}

1) Bicakcigil M, Aksu K, Kamali S, Ozbalkan Z, Ates A, Karadag O, Ozer HT, Seyahi E, Akar S, Onen F, Cefle A, Aydin SZ, Yilmaz N, Onat AM, Cobankara V, Tunc E, Ozturk MA, Fresko I, Karaaslan Y, Akkoc N, Yucel AE, Kiraz S, Keser G, Inanc M and Direskeneli H: Takayasu's arteritis in Turkey - clinical and angiographic features of 248 patients. Clin Exp Rheumatol, 2009; 27: S59-64

2) Kerr GS, Hallahan CW, Giordano J, Leavitt RY, Fauci AS, Rottem M and Hoffman GS: Takayasu arteritis. Ann Intern Med, 1994; 120: 919-929

3) Andrews J and Mason JC: Takayasu's arteritis--recent 
advances in imaging offer promise. Rheumatology (Oxford), 2007; 46: 6-15

4) Soto ME, Espinola N, Flores-Suarez LF and Reyes PA: Takayasu arteritis: clinical features in 110 Mexican Mestizo patients and cardiovascular impact on survival and prognosis. Clin Exp Rheumatol, 2008; 26: S9-15

5) Park MC, Lee SW, Park YB, Chung NS and Lee SK: Clinical characteristics and outcomes of Takayasu's arteritis: analysis of 108 patients using standardized criteria for diagnosis, activity assessment, and angiographic classification. Scand J Rheumatol, 2005; 34: 284-292

6) Lee GY, Jang SY, Ko SM, Kim EK, Lee SH, Han H, Choi $\mathrm{SH}$, Kim YW, Choe YH and Kim DK: Cardiovascular manifestations of Takayasu arteritis and their relationship to the disease activity: analysis of 204 Korean patients at a single center. Int J Cardiol, 2012; 159: 14-20

7) Vlachopoulos C, Aznaouridis K and Stefanadis C: Prediction of cardiovascular events and all-cause mortality with arterial stiffness: a systematic review and meta-analysis. J Am Coll Cardiol, 2010; 55: 1318-1327

8) Yamashina A, Tomiyama H, Arai T, Hirose K, Koji Y, Hirayama Y, Yamamoto Y and Hori S: Brachial-ankle pulse wave velocity as a marker of atherosclerotic vascular damage and cardiovascular risk. Hypertens Res, 2003; 26: 615-622

9) Riggio S, Mandraffino G, Sardo MA, Iudicello R, Camarda N, Imbalzano E, Alibrandi A, Saitta C, Carerj S, Arrigo T and Saitta A: Pulse wave velocity and augmentation index, but not intima-media thickness, are early indicators of vascular damage in hypercholesterolemic children. Eur J Clin Invest, 2010; 40: 250-257

10) Xiong Z, Zhu C, Zheng Z, Wang M, Wu Z, Chen L and Chen Y: Relationship between arterial stiffness assessed by brachial-ankle pulse wave velocity and coronary artery disease severity assessed by the SYNTAX score. Journal of atherosclerosis and thrombosis, 2012; 19: 970-976

11) Ohkuma T, Ninomiya $T$, Tomiyama $H$, Kario K, Hoshide S, Kita Y, Inoguchi T, Maeda Y, Kohara K, Tabara Y, Nakamura M, Ohkubo T, Watada H, Munakata M, Ohishi M, Ito N, Nakamura M, Shoji T, Vlachopoulos C, Yamashina A and Collaborative Group for JB: BrachialAnkle Pulse Wave Velocity and the Risk Prediction of Cardiovascular Disease: An Individual Participant Data Meta-Analysis. Hypertension, 2017; 69: 1045-1052

12) Kim M, Kim M, Yoo HJ, Lee SY, Lee SH, Lee JH: AgeSpecific Determinants of Pulse Wave Velocity among Metabolic Syndrome Components, Inflammatory Markers, and Oxidative Stress. J Atheroscler Thromb, 2018; 25: $178-185$

13) Mozos I, Malainer C, Horbanczuk J, Gug C, Stoian D, Luca CT and Atanasov AG: Inflammatory Markers for Arterial Stiffness in Cardiovascular Diseases. Front Immunol, 2017; 8: 1058

14) Ng WF, Fantin F, Ng C, Dockery F, Schiff R, Davies KA, Rajkumar C and Mason JC: Takayasu's arteritis: a cause of prolonged arterial stiffness. Rheumatology (Oxford), 2006; 45: 741-745

15) Salles Rosa Neto N, Levy-Neto M, Tolezani EC, et al.: Determinants of arterial stiffness in female patients with Takayasu arteritis. J Rheumatol, 2014; 41: 1374-1378

16) Arend WP, Michel BA, Bloch DA, Hunder GG, Calabrese
LH, Edworthy SM, Fauci AS, Leavitt RY, Lie JT, Lightfoot RW, Jr. and et al.: The American College of Rheumatology 1990 criteria for the classification of Takayasu arteritis. Arthritis Rheum, 1990; 33: 1129-1134

17) Yamashina A, Tomiyama $H$, Takeda $K$, Tsuda $H$, Arai $T$, Hirose K, Koji Y, Hori S and Yamamoto Y: Validity, reproducibility, and clinical significance of noninvasive brachial-ankle pulse wave velocity measurement. Hypertens Res, 2002; 25: 359-364

18) Tsuchikura S, Shoji T, Kimoto E, Shinohara K, Hatsuda $\mathrm{S}$, Koyama $\mathrm{H}$, Emoto $\mathrm{M}$ and Nishizawa Y: Brachial-ankle pulse wave velocity as an index of central arterial stiffness. Journal of atherosclerosis and thrombosis, 2010; 17: 658665

19) Wang $X$ and Dang A: Prognostic Value of Brachial-Ankle Pulse Wave Velocity in Patients With Takayasu Arteritis With Drug-Eluting Stent Implantation. Arthritis Care Res (Hoboken), 2015; 67: 1150-1157

20) McEniery CM and Wilkinson IB: Large artery stiffness and inflammation. J Hum Hypertens, 2005; 19: 507-509

21) Libby P, Ridker PM and Maseri A: Inflammation and atherosclerosis. Circulation, 2002; 105: 1135-1143

22) Maki-Petaja KM, Hall FC, Booth AD, Wallace SM, Yasmin, Bearcroft PW, Harish S, Furlong A, McEniery CM, Brown J and Wilkinson IB: Rheumatoid arthritis is associated with increased aortic pulse-wave velocity, which is reduced by anti-tumor necrosis factor-alpha therapy. Circulation, 2006; 114: 1185-1192

23) Yildiz M, Yildiz BS, Soy M and Tutkan H: Impairment of arterial distensibility in premenopausal women with systemic lupus erythematosus. Kardiol Pol, 2008; 66: 11941199; discussion 1200-1191

24) Yildiz M, Soy M, Kurum T and Yildiz BS: Arterial distensibility in Wegener's granulomatosis: a carotid-femoral pulse wave velocity study. Anadolu Kardiyol Derg, 2007; 7: 281-285

25) Gisondi P, Fantin F, Del Giglio M, Valbusa F, Marino F, Zamboni $\mathrm{M}$ and Girolomoni G: Chronic plaque psoriasis is associated with increased arterial stiffness. Dermatology, 2009; 218: 110-113

26) Yildiz M, Biteker M and Ozkan M: Assessment of aortic stiffness and ventricular functions in familial Mediterranean fever. Anadolu Kardiyol Derg, 2008; 8: 395; author reply 395-396

27) Yildiz M: Arterial distensibility in chronic inflammatory rheumatic disorders. Open Cardiovasc Med J, 2010; 4: 83-88

28) Chang HK, Kim SK, Lee SS and Rhee MY: Arterial stiffness in Behcet's disease: increased regional pulse wave velocity values. Ann Rheum Dis, 2006; 65: 415-416

29) Cypiene A, Laucevicius A, Venalis A, Dadoniene J, Ryliskyte L, Petrulioniene Z, Kovaite M and Gintautas J: The impact of systemic sclerosis on arterial wall stiffness parameters and endothelial function. Clin Rheumatol, 2008; 27: 1517-1522

30) Direskeneli H, Aydin SZ and Merkel PA: Assessment of disease activity and progression in Takayasu's arteritis. Clin Exp Rheumatol, 2011; 29: S86-91

31) Direskeneli H: Clinical assessment in Takayasu's arteritis: major challenges and controversies. Clin Exp Rheumatol, 2017; 35 Suppl 103: 189-193 
32) Booth AD, Wallace S, McEniery CM, Yasmin, Brown J, Jayne DR and Wilkinson IB: Inflammation and arterial stiffness in systemic vasculitis: a model of vascular inflammation. Arthritis Rheum, 2004; 50: 581-588

33) Maki-Petaja KM, Elkhawad M, Cheriyan J, Joshi FR, Ostor AJ, Hall FC, Rudd JH and Wilkinson IB: Antitumor necrosis factor-alpha therapy reduces aortic inflammation and stiffness in patients with rheumatoid arthritis. Circulation, 2012; 126: 2473-2480

34) Dulai R, Perry M, Twycross-Lewis R, Morrissey D, Atzeni $\mathrm{F}$ and Greenwald S: The effect of tumor necrosis factor- alpha antagonists on arterial stiffness in rheumatoid arthritis: a literature review. Semin Arthritis Rheum, 2012; 42: 1-8

35) Lupi-Herrera E, Sanchez-Torres G, Marcushamer J, Mispireta J, Horwitz S and Vela JE: Takayasu's arteritis. Clinical study of 107 cases. Am Heart J, 1977; 93: 94-103

36) Hoffman GS: Takayasu arteritis: lessons from the American National Institutes of Health experience. Int J Cardiol, 1996; 54 Suppl: S99-102 